Société d'histoire de la révolution de 1848 et des

révolutions du XIXe siècle

48 | 2014

Usages du droit

\title{
Le « pluralisme juridique ». Au fil d'un conflit de succession en Méditerranée à la fin du XIX ${ }^{\mathrm{e}}$ siècle
}

A concept seen through an inheritance conflict in late nineteenth-century central Mediterranean

Der „Rechtspluralismus“. Ein Nachfolgekonflikt im Mittelmeerraum am Ende des 19. Jahrhunderts

M'hamed Oualdi

\section{(2) OpenEdition}

Journals

Édition électronique

URL : http://journals.openedition.org/rh19/4659

DOI : $10.4000 /$ rh 19.4659

ISSN : $1777-5329$

Éditeur

La Société de 1848

Édition imprimée

Date de publication : 1 juin 2014

Pagination : 93-106

ISSN : 1265-1354

Référence électronique

M'hamed Oualdi, «Le «pluralisme juridique ». Au fil d'un conflit de succession en Méditerranée à la fin du XIXe siècle », Revue d'histoire du XIXe siècle [En ligne], 48 | 2014, mis en ligne le 17 septembre 2014 consulté le 10 décembre 2020. URL : http://journals.openedition.org/rh19/4659; DOI : https://doi.org/ $10.4000 /$ rh19.4659 


\section{M'HAMED OUALDI}

\section{Le "pluralisme juridique». Au fil d'un conflit de succession en Méditerranée à la fin du XIX siècle $^{1}$}

La succession du général Husayn, parce qu'elle met en jeu des systèmes juridiques concurrents aux ramifications complexes, permet d'aborder un cas de pluralisme juridique spécifique aux situations de transition coloniale. L'origine de l'affaire tient au statut de Husayn : affranchi d'origine circassienne, éduqué et promu auprès des gouverneurs de la province ottomane de Tunis jusqu'à se voir confier la présidence de la municipalité de la capitale en 1860, puis la charge de ministre de l'éducation au cours des années $1870^{2}$. Sitôt prévenu du décès du général, le 27 juin 1887, le consul ottoman à Florence, Musurus Ghikis, fit apposer des scellés dans la villa et l'appartement occupés par le défunt dans la capitale toscane ${ }^{3}$. Première autorité informée du trépas du dignitaire "tunisien", le consul qui considérait le défunt avant tout comme un sujet ottoman avait devancé toute action du représentant français alors que depuis l'instauration du protectorat français sur la Tunisie en 1881, les agents diplomatiques français étaient "chargés de la protection des intérêts tunisiens et des nationaux de la régence» de Tunis ${ }^{4}-$ ancienne province ottomane du Maghreb.

Quelques jours plus tard, début juillet 1887, l'affaire connut son premier rebondissement : les autorités françaises découvrirent que Husayn prévoyait, dans un testament de léguer un tiers de son patrimoine à deux jeunes filles — ses filles illégitimes - à condition que, parvenues à l'âge adulte, elles épousent des musulmans. Pourtant, selon les normes musulmanes, le général ne disposait pas librement de sa succession : en l'absence de toute descendance reconnue, ses biens d'affranchi devaient revenir à son maitre ou à l'héritier reconnu de ce patron, à savoir à cette date, 'Alī Bey qui n'était autre

1. Cet article doit beaucoup aux conseils et relectures de Franck-Alexandre Lecomte et Guillaume Calafat, qu'ils en soient ici remerciés.

2. M'hamed Oualdi, Esclaves et maitres. Les mamelouks au service des beys de Tunis du XVII siècle aux années 1880, Paris, Publications de la Sorbonne, 2011, p. 247-250, 396-397.

3. ANT (Archives nationales tunisiennes), C. (Carton) 11, d. (dossier) 113, doc. 8762, David Santillana, Florence, le $1^{\text {er }}$ juillet 1887.

4. Article 6 du traité du Bardo, 12 mai 1881. ANT, C. 11, d. 113b, doc. 8959, Santillana au résident Massicault, Florence, 2 février 1888. 
que le gouverneur de Tunisie, maintenu par la nouvelle autorité française. Pour 'Alī Bey, le général n'ayant pas reconnu les deux filles à qui il voulait léguer une partie de sa fortune, il n'était pas légalement en mesure de leur transmettre des biens.

Par cet imprévu testamentaire, le conflit de succession se compliquait d'affaires privées et publiques impliquant le gouverneur de Tunis, les mères des deux jeunes filles (l'une italienne, l'autre d'origine allemande) et un nombre toujours croissant d'experts, d'intermédiaires et de plaignants, anciens employés du gouvernement tunisien et créanciers du défunt général, installés en Toscane, venus de Tunis, de l'Algérie coloniale ou liés à des milieux d'affaires français, égyptiens voire britanniques.

Le lieu du décès, Florence, poussa des acteurs à recourir aux juridictions italiennes, sans pour autant situer l'affaire dans un système judiciaire hiérarchisé et cohérent car d'une part, dans les affaires de succession, le droit italien et le droit international privé en général, prenaient en considération le droit d'origine du défunt ${ }^{5}$. D'autre part, les conflits soulevés en Toscane autour de l'héritage du général Husayn étaient liés à d'autres litiges formulés devant d'autres tribunaux à Alexandrie ${ }^{6}$, et surtout auprès de tribunaux de France et de Tunisie 7 . Une grande partie des biens fonciers du défunt se concentrait autour de Tunis, suscitant des contestations de créanciers ou de locataires, et tout appel d'un jugement de tribunaux français installés à Tunis depuis 1883 se faisait auprès de la cour $\mathrm{d}^{\prime}$ Alger ${ }^{8}$. En outre, le général Husayn avait représenté les intérêts de son gouvernement dans une affaire de succession autrement plus considérable, celle du caïd Nissim Scemama, directeur des Finances tunisiennes qui avait fui vers la France en 1864, puis en Toscane, en détournant près de 20 millions de francs du Trésor tunisien.

Pour toutes ces raisons, et parce qu'ils se méfiaient de leurs homologues italiens ${ }^{9}$, les représentants des autorités françaises s'accordèrent assez vite sur la nécessité de transporter tous les dossiers de cette affaire vers les tribunaux de Tunis ${ }^{10}$. Une fois présentés en Tunisie, à partir du début des années 1890 , les multiples litiges liés à ce conflit de succession pouvaient être soumis à diverses institutions : tribunaux français placés sous la tutelle de la haute

5. ANT, C. 11, d. 113, doc. 8762 , David Santillana, Florence, $1^{\text {er }}$ juillet 1887 ; C. 11, d. 113, doc. 8786, consulat de France à Florence au Secrétaire général, Régnault, Florence, 15 juillet 1887.

6. ANT, C. 11, d. 98, doc. 90, résidence générale au consul de France à Alexandrie, mai 1891; ANT, C. 11, d 103, doc. 7729, al-Hādī Zarrūq au Premier ministre, Bū 'Attūr, 17 juillet 1887.

7. Ali Noureddine, "Opposants et serviteurs : les deux faces de la magistrature française dans la Régence de Tunis (1883-1886)", Contributions du séminaire sur les administrations coloniales (20092010), Paris, IHTP, 2011, p. 21.

8. Mary Dewhurst Lewis, 'Geographies of Power : The Tunisian Civic Order, Jurisdictional Politics, and Imperial Rivalry in the Mediterranean, 1881-1935', The Journal of Modern History, vol. 80, n 4 , p. 804, 2008.

9. ANT, lettre anonyme adressée à « un ministre», Tunis, le 17 janvier $1888, \mathrm{f}^{\circ} 1 \mathrm{r}$.

10. ANT, C. 11, d. 113b, doc. 9096, avocat Santillana à Régnault, secrétaire général, Florence, 28 janvier 1888 . 
cour d'Alger ${ }^{11}$; conseil dit «charaïque» de juges musulmans ${ }^{12}$ voire tribunaux rabbiniques en charge des affaires de statut personnel. En ces années 18801900 - et c'est un point fondamental -, les services français contrôlaient des pans entiers de ce système judiciaire tunisien, mais aucune hiérarchie ne le structurait de manière formelle. Les juridictions poursuivaient des accusés selon la sujétion, la confession et la nationalité des uns et des autres. Elles disposaient de leurs propres systèmes d'appel. Et l'ordre judiciaire colonial pouvait lui même être travaillé par les normes locales ${ }^{13}$. La pluralité des terrains juridiques connectés dans cette affaire concorde de fait avec les définitions pour lesquelles il y a pluralisme juridique lorsque «deux systèmes légaux au moins coexistent dans un même espace social $»^{14}$. Mais, sans se contenter de fondre ce cas dans une définition taillée sur mesure, c'est l'outil analytique $\mathrm{du}$ "pluralisme juridique» que nous voulons interroger au travers de l'étude de ce conflit de succession.

Ce concept dont les racines plongent au cœur du XIX ${ }^{\mathrm{e}}$ siècle dans des pensées évolutionnistes puis fonctionnalistes du droit, est formalisé dans les années 1970 et devient dans le même temps objet de débat pour les études du droit dans les espaces coloniaux et postcoloniaux ${ }^{15}$. À partir des années 1980 et plus clairement dans les années 1990, il n'a cessé d'être remis en cause surtout dans les champs du droit et de l'anthropologie ${ }^{16}$. Dans cette contribution, il ne s'agira pas de se positionner pour ou contre la notion de pluralisme juridique, mais d'abord de comprendre comment ce concept, ainsi que les amendements qui lui ont été apportés, peuvent aider à historiciser des rapports de force formulés par le droit. Jusqu'à quel point l'accentuation de rivalités coloniales et le chevauchement des autorités impériales se cristallisent-ils autour du juridique, aboutissant à un recours au droit?

Dans une seconde étape, nous percevons quels types d'ordre ou de désordre résultent des initiatives d'acteurs placés en situation de pluralisme juridique. L'historienne du droit, Lauren Benton, y voit des dynamiques de constructions d'ordres juridiques coloniaux initiés par les actions des particuliers et entretenus par les réponses d'autorités administratives ${ }^{17}$, tandis que Paolo Sartori percevait à l'inverse la diffusion de la connaissance juridique

11. ANT, C. 11, d. 98 doc. 18, huissier Carnol, Alger, 4 janvier 1890.

12. ANT, C. 11, d 103, doc. 8823, lettre du cadi al-Nayfar, au Premier ministre Bū 'Attūr, 11 septembre 1887 .

13. Paolo Sartori, Ido Shahar, 'Legal Pluralism in Muslim-Majority Colonies: Mapping the Terrain', Journal of the Economic and Social History of the Orient, $\mathrm{n}^{\circ}$ 55, 2012, p. 638.

14. Sally Engle Merry, 'Legal Pluralism', Law \& Society Review, volume 22, n 5, 1988, p. 870.

15. Franz von Benda-Beckmann, 'Who's afraid of Legal Pluralism', Journal of Legal Pluralism and Unofficial Law, n 47, 2002, p. 16; Sally Engle Merry, 'Legal Pluralism', art. cit., p. 869; Beaudoin Dupret, 'Legal Pluralism, Plurality of Laws and Legal Practices: Theories, Critiques, and Praxiological Re-specification', European Journal of Legal Studies, 1, 1, 2007, p. 2, 12.

16. Chris Fuller, 'Legal Anthropology: Legal Pluralism and Legal Thought', Anthropology Today, volume $10, n^{\circ} 3,1994$, p. 9-12.

17. Lauren Benton, 'Colonial Law and Cultural Difference: Jurisdictionnal Politics and the Formation of the Colonial State', Comparative Studies in Society and History, vol. 41, n 3, juillet 1999, p. $563-588$. 
comme une source de conflits juridiques et de fragmentation sociale de l'ordre colonial ${ }^{18}$. Or, l'étude de cas ici abordée, en ne se limitant pas au seul cadre colonial ou national, éclaire le jeu des dynamiques individuelles entre ces divers champs juridiques connectés. Enfin, à suivre le concept de pluralisme juridique, à penser que le pluralisme est partout, que tout espace social est marqué par un pluralisme normatif, en une troisième étape plus limitée, nous nous demanderons quelles autres perspectives peuvent être saisies audelà du juridique afin d'appréhender la succession du général Husayn?

$*$

\section{INTENSIFICATION DES CONFLITS ET CATÉGORISATION D'UN « DROIT MUSULMAN"}

Si ce cas d'étude est le pur produit d'une situation de transition impériale, il ne constitue pourtant pas un conflit de succession à part. L'inclusion d'enfants non reconnus ou illégitimes dans un testament n'est en effet pas propre à cette affaire. De même, l'action rendue possible à partir d'un espacetiers - l'Italie en l'occurrence - n'est pas singulier : le recours au droit international a été certes, plus fréquent aux temps coloniaux "pour régler les relations entre nations impériales ${ }^{19}$, mais si l'on s'en tient à l'échelle d'analyse retenue ici, un espace juridique qui comprendrait la Toscane, la région de Tunis et une extension à Alger, l'on constate que dès le XVI ${ }^{e}$ siècle, les tribunaux de Toscane et les tribunaux consulaires français en Afrique du Nord statuaient déjà sur des affaires impliquant des figures de l'autorité à Tunis et Alger. Il faut ici rappeler le rôle prégnant du port franc de Livourne dans les échanges entre l'Europe latine et les provinces ottomanes du Maghreb ${ }^{20}$.

Pour saisir la dynamique particulière de la succession du général Husayn, il faut la confronter avec deux autres affaires impliquant deux dignitaires de l'ancienne province de Tunis : celle de la succession du caïd Nissim Scemama, déjà évoquée, et celle du général Mahmoud Benayad qui s'enrichit en cumulant fermes d'État et fonctions gouvernementales avant de s'installer à Paris en 1852, puis à Istanbul en $1857^{21}$. Dans ces cas, des fonds publics et patrimoniaux de gouverneurs de Tunis sont dirigés et souvent détournés par des serviteurs de ces gouverneurs vers des circuits financiers européens, avant tout vers des banques françaises et italiennes. Dans les trois cas, le gouvernement

18. Paolo Sartori, «Premessa. Waqf, colonialismo e pluralismo giuridico nelle società musulmane», Quaderni storici, $\mathrm{n}^{\circ} 132,2009$, p. 627-652.

19. Emmanuelle Saada, "Citoyens et sujets de l'empire français : les usages du droit en situation coloniale», Genèses, n' 53,2003 , p. 8.

20. Guillaume Calafat, "Les frontières du droit en Méditerranée. Marchands et marins face aux tribunaux maritimes (1570-1670) » in Albrecht Fuess et Bernard Heyberger [dir.], La frontière méditerranéenne. Échanges, circulations et affrontements, Turnhout, Brepols (à paraitre).

21. Jean Ganiage, Les origines du protectorat français en Tunisie (1861-1881), Tunis, MTE, Paris, PUF, 1959, p. 572, 600-601. 
de Tunis est amené à défendre ses intérêts devant des juridictions nationales variées. Et à chaque fois, c'est le statut juridiquement incertain des acteurs centraux de ces affaires qui permet des actions judiciaires en plusieurs lieux et en usant de multiples répertoires normatifs : une position d' "exilé» pour le général Husayn qui, au lendemain de la conquête française, ne bénéficie plus d'aucune charge du gouvernement tunisien; des basculements d'allégeance dans le cas du général Benayad naturalisé français après sa fuite en 1852 et dans celui de Nessim Scemama, anobli par le roi d'Italie en 1866.

Ces trois cas qui s'échelonnent dans la seconde moitié du XIX siècle ne sont pas suivis, à notre connaissance, d'autres équivalents, dans l'espace d'analyse concerné. Cela bien sûr ne veut pas dire qu'à partir des années 1890, d'autres affaires impliquant des gens de Tunis voire d'Alger n'aient pas été examinées par les juridictions toscanes. Mais rapprochés les uns des autres, ces trois cas exposent deux inflexions dans les dynamiques régionales du pluralisme juridique. Ces affaires n'impliquent pas seulement deux champs juridiques ou plus précisément deux territoires judiciaires (en l'occurrence la région de Tunis et la Toscane) mais des cours d'autres pays européens notamment des tribunaux français ${ }^{22}$. Et, surtout, les intérêts d'un gouvernement (en l'occurrence tunisien) sont non seulement pour partie dépersonnalisés, mais deviennent aussi l'enjeu de rivalités diplomatiques.

Outre le contexte diplomatique de transformations impériales, deux phénomènes majeurs plus profonds ont contribué à un raidissement des pratiques et des appréhensions du pluralisme juridique. Le premier phénomène est celui d'une intégration financière plus poussée dans la seconde moitié du XIX ${ }^{\mathrm{e}}$ siècle entre les institutions gouvernementales de Tunis (et d'autres capitales de l'Empire ottoman) et les banques européennes. Afin de financer la mise en place d'armées régulières et la montée en puissance de leurs administrations, des autorités dont celles de Tunis ont contracté des prêts sur des places européennes. Les conditions désavantageuses de ces prêts et la gestion patrimoniale de ces finances publiques par la dynastie de gouverneurs en place à Tunis, ont abouti à des pressions fiscales plus contraignantes sur des sujets de cette province ottomane. À partir des années 1860, la dette publique a été financée par une hausse des impôts. En conséquence, de plus en plus de Tunisiens ont été contraints à contracter des crédits pour payer des taxes diverses ou pour régler des amendes collectives. Les cas Benayad, Scemama, et Husayn dans une moindre mesure, traduisent cette connexion croissante des circuits financiers entre les deux rives de la Méditerranée et les revendications plus fortes autour des patrimoines et des fonds publics d'un territoire politique convoitée ${ }^{3}$.

22. ANT, C. 107, doc. 136, 7 février 1874, «assignation de l'ancien Premier ministre de Tunis, Mustafa Khaznadar par Mahmoud Ben Aïad, devant tribunal de commerce de la Seine à propos d'une association pour l'achat en Europe et la vente de marchandises et toutes autres opérations commerciales".

23. Didier Guignard, L'abus de pouvoir dans l'Algérie coloniale, Paris, Presses universitaires de Paris-Ouest, 2011. 
Mais ces biens et finances accaparés et parfois détournés ne poseraient pas tant de problèmes si ceux qui étaient réputés les posséder et les léguer, Mahmoud Benayad, Nessim Scemama et Husayn, n'avaient pas prêté allégeance à des autorités politiques diverses et souvent concurrentes. Dans la seconde moitié du XIX ${ }^{\mathrm{e}}$ siècle, le phénomène très parlant de la protection s'est ainsi développé à travers l'ensemble du Maghreb non colonisé et dans les composantes de l'Empire ottoman. Les protégés étaient à l'origine des agents des consuls ou de marchands européens dépendant juridiquement et fiscalement d'autorités consulaires européennes. L'intérêt financier de ce type de statut n'échappa pas aux notables locaux, si bien que certains optèrent pour cette forme d'allégeance qui les plaçait dans un entre-deux, servant un pouvoir et ne rendant compte qu'à une autre autorité diplomatique. Le général Husayn relevait cependant d'un autre cas de figure : se percevant, dans sa correspondance, comme un agent des sultans ottomans, et se concevant comme un défenseur du monde musulman contre les "Francs » ${ }^{24}$, le général perdit tout lien avec le gouvernement tunisien quand des doutes furent émis sur la reddition de ses comptes au terme de sa mission en Toscane. Resté en Italie, ne se résignant pas à finir sa vie à Istanbul auprès d'exilés tunisois, la condition du général se rapprochait de celle d'un apatride au sens où il ne pouvait être clairement identifié ni comme un sujet ottoman, ni comme un sujet tunisien "protégé " par l'État colonial français.

Ce cas, tout comme celui des protégés, plaçait les hommes et leurs patrimoines à des carrefours de juridiction qui ont contribué à une cristallisation de revendications juridiques, mais ce recours privilégié au juridique a aussi été rendu possible par une autre dynamique, celle du champ normatif musulman qui commençait, sous l'influence des Européens, à être défini et essentialisé en "droit musulman", masquant de ce point de vue des polysémies et des différences d'usage ${ }^{25}$. Dans un processus comparable à celui discerné par Edward Saïd d'invention d'un Orient par l'Occident, les tenants de la thèse d'une invention d'un droit musulman - surtout par les juristes des institutions coloniales - se fondent sur toute une série de mutations et d'indices tels que la transplantation rigidifiée de catégories juridiques voire de normes issues de l'Europe et de la France postrévolutionnaire. Ainsi, la propriété individuelle se substitue à une multiplicité de régimes reconnus de l'appropriation ${ }^{26}$, et le mariage civil remplace un processus gradué de l'alliance matrimoniale ${ }^{27}$. Sont aussi mis en avant la transformation du régime de la preuve (qui, en contexte musulman, était fondé sur le témoignage oral plus que sur l'écrit), la codification de coutumes perdant de leur plasticité

24. Général Husayn, Lettres du Général Hussein à Khérédine (XIXe siècle), Carthage, Bayt al-Hikma, 1992, tome 2, lettre 159, 12 mai 1877, p. 126.

25. Sally Engle Merry, 'Legal Pluralism', art. cit., p. 871.

26. Isabelle Grangaud, "Prouver par l'écriture : propriétaires algérois, conquérants français et historiens ottomanistes", Genèses, n 74, mars 2009, p. 25-45.

27. Nawel Gafsia, L'invention coloniale du mariage tunisien : le cas tunisien, Paris, LGDJ, 2008. 
une fois écrites ${ }^{28}$, les procédures de centralisation et de standardisation ${ }^{29}:$ en somme la «traditionalisation" de ce qui était auparavant labile et ne devait pas se comprendre par référence à une langue européenne, par traduction de documents en cette langue ${ }^{30}$. Un des agents centraux de la résolution du conflit autour de la succession du général Husayn, le juriste David Santillana pourrait d'ailleurs être perçu comme un des acteurs de la constitution d'un "droit musulman». Né à Tunis, arabisant, nourri de culture italienne, Santillana fut non seulement choisi par les autorités françaises pour représenter le gouverneur de Tunis dans l'affaire Husayn, mais par la suite, il fut aussi appelé à siéger dans la commission de codification des lois tunisiennes et connu comme auteur d'une somme sur le «droit musulman malékite», selon le nom d'une des écoles d'interprétation de ce droit ${ }^{31}$.

Ce qui nous semble plus fondamentalement en jeu dans ce double processus (de mise en conformité d'un champ normatif et de connexions accrues entre circuits financiers, allégeances politiques et hiérarchies judiciaires), c'est la valorisation ou à l'inverse l'abandon de territorialités juridiques, au sens des territoires de pratiques et de recours aux institutions judiciaires ${ }^{32}$. Un des derniers facteurs de raidissement et de cristallisation autour du droit fut la question des lieux d'arbitrage. Dans l'affaire de la succession du général Husayn, la hiérarchie politique ottomane se retire très vite du conflit, dès septembre-octobre 1887 , et renonce rapidement à toute prétention formelle ${ }^{33}$. La Toscane se maintient un temps comme un espace de recours, mais parallèlement l'importance de l'espace juridique lié à la métropole française se confirme. Tout l'enjeu pour la diplomatie française est de recourir à des experts obéissant à une hiérarchie française mais surtout de transporter l'inventaire de la succession Husayn et les conflits qui y sont liés vers la Tunisie, contribuant à la constitution d'un espace juridique hiérarchisé à l'échelle de ce pays. À cette étape de la réflexion, se pose la seconde question : celle des conséquences de la concurrence des droits, de ses effets, au regard des actions et des conceptions des particuliers et des institutions.

28. Paul Molinier, L'islamologie juridique française durant la période coloniale, mémoire de master 2, EPHE, dir. Mohammed H. Benkheira, 2010. p. 643 .

29. Cas du Mejelle ottoman en 1876, Paolo Sartori, Ido Shahar, 'Legal Pluralism...', art. cit.,

30. Dipesh Chakrabarty, Provincializing Europe. Postcolonial Thought and Historical Difference, Oxford, Oxford University Press, 2001, p. 5-6.

31. Frederico Cresti, «Entre connaissance scientifique et politique coloniale. Les orientalistes italiens et les pays de la Méditerranée islamique entre la fin du XIX et les premières décennies du XX' siècle", in Benoît Grévin [dir.], Maghreb-Italie. Des passeurs médiévaux à l'orientalisme moderne (XIII'-milieu XXe siècle), Rome, École française de Rome, 2010, p. 400-403.

32. Alain Supiot, «L'inscription territoriale des lois», Esprit, novembre 2008, p. 151-170.

33. ANT, C. 11, d. 113, doc. 8824, Khayr al-Din à 'Alī Bey, 18 septembre 1887. 


\section{CE QU'ACTEURS ET INSTITUTIONS FONT}

D'UNE SITUATION DE PLURALISME

Selon cette seconde approche, à quel type de construction ou de déconstruction du social et du politique les acteurs aboutissent-ils? Les analyses qui mettent avant tout l'accent soit sur la toute puissance des institutions ou à l'inverse sur la capacité des acteurs à jouer des failles entre institutions ne peuvent être satisfaisantes ${ }^{34}$. Un des acteurs de l'affaire Husayn, Léon Elmilik, juif d'Algérie et ancien bras droit du général, pourrait en effet illustrer ce type de stratégie par sa capacité à pousser à bout les autorités françaises et à obtenir une compensation financière non négligeable. Cependant, si ces analyses permettent d'affiner la compréhension des processus de domination, elles n'aident pas à resituer les acteurs et institutions dans des dynamiques et des constructions historiques plus larges.

De ce point de vue, une des grilles d'analyse les plus stimulantes est celle forgée par Lauren Benton à la fin des années 1990. Celle-ci prend à la fois en compte la force institutionnelle et l'agilité potentielle des acteurs. Son schéma d'interprétation propose de concevoir les mutations des ordres juridiques pluriels en situation coloniale à partir des interactions entre plaignants, représentants des institutions coloniales et médiateurs. Selon cette vision, la construction d'un ordre colonial, à partir d'une phase de relative fluidité du pluralisme juridique vers un modèle plus hiérarchisé, a procédé par à-coups et en réponse à une myriade de conflits autour des définitions des différences culturelles, de propriété et d'autorité morale. Dans ce schéma, ce qui a permis de renforcer le «droit étatique» (State law), c'est le fait que des acteurs aient fait appel à son autorité. De fait, pour Lauren Benton, il devient plus intéressant de prêter attention aux marques de ces luttes sur les institutions que d'évaluer les succès et échecs des manœuvres juridiques des colonisés ${ }^{35}$.

Les traits majeurs de la conclusion du conflit autour de la succession du général Husayn iraient ainsi dans le sens d'une construction d'un ordre colonial à travers les litiges impulsés par des particuliers et médiateurs. Face aux nombreux procès intentés en Toscane, les autorités françaises sont ainsi parvenues à «rapatrier ", à partir de 1889 , les papiers de l'inventaire et les conflits qui lui sont liés dans un territoire juridictionnel tunisien, puis à replacer la succession entre les mains du gouverneur de Tunis. Cependant, ne penser qu'en terme d'homogénéisation ne suffit pas à comprendre ce cas transnational car, d'un autre côté, ces autorités françaises ne parviennent à ce résultat qu'en multipliant les compromis avec les différentes figures de l'affaire. De fait, une explication trop dialectique sous-estimerait ou mettrait de côté

34. Julia Clancy-Smith, 'Women, Gender and Migration along a Mediterranean Frontier: PreColonial Tunisia, c. 1815-1870', Gender and History, n 17, 2005, p. 77.

35. Lauren Benton, 'Colonial Law...', art. cit., p. 563-564, p. 588. 
la persistance, l'agrégation ou l'intensification des formes de contestation des ordres coloniaux. Dans une introduction à un dossier sur des biens de mainmorte (waqf, habous) aux temps coloniaux, l'historien de l'Asie centrale contemporaine, Paolo Sartori favorisait au contraire l'idée d'un "accroissement des conflits sociaux, de recherche de réglementation" et de «formulation juridique des relations entre musulmans", du fait de la coexistence de droits divers et de la "bureaucratisation de la pratique juridique »"

Les deux modes d'interprétation présentés ici brièvement et schématiquement, l'un de construction d'un ordre par la conflictualité et le second de diffusion des conflits par l'expansion d'un langage juridique, ont pour point commun de pointer ce qui est aux fondements de diverses stratégies juridiques, à savoir les registres de connaissance et d'appréhension du droit et des droits. En deçà de ces problématiques, le pluralisme juridique peut en effet aussi être saisi selon les propositions de l'anthropologue du droit, Beaudoin Dupret, selon une approche praxiologique, une analyse fondée "sur les orientations» et "les caractérisations des gens", selon l'idée que la loi est d'abord «déterminée par les gens, par leurs usages dans l'arène sociale » ${ }^{37}$. Dans le cas qui nous intéresse, ce choix méthodologique permettrait de saisir la perception qu'une partie de ces gens avait du droit, de leurs capacités plus ou moins grandes à distinguer des champs juridiques, pour discerner et éventuellement concilier des dynamiques forts différentes impulsées par la concomitance de différents systèmes juridiques.

Parmi les acteurs des conflits autour de la succession Husayn, nombre d'entre eux ont dû se déplacer entre la Toscane, Tunis, parfois Alger, Le Caire, Istanbul, les principales capitales européennes de l'époque (Paris, Londres), voire New York (pour la mère d'une des jeunes filles à qui était promis un sixième de l'héritage). Dans leur déplacement, il n'est pas évident que tous soient parvenus à établir des distinctions entre des institutions judiciaires voire entre des champs juridiques, et plus généralement encore entre des champs normatifs. Les cœurs peuvent difficilement être sondés mais l'hypothèse d'une notion confuse et polyvalente de ce qui est juste est certainement à prendre en compte. De même, il ne faut pas sous estimer la capacité des hommes de droit, avocats, conseillers, à insuffler à leurs clients un métalangage juridique et des manières d'identifier et de hiérarchiser les institutions ${ }^{38}$.

Cependant, parmi ceux qui ont le plus été à même d'agir dans ce cas, des agents de l'administration française jusqu'aux conseillers de ces autorités, la conscience d'une distinction des champs juridiques était plus évidente. Les

36. Paolo Sartori, «Premessa. Waqf, colonialismo...», art. cit., p. 643.

37. Beaudoin Dupret, 'Legal Pluralism, Plurality of Laws...', art. cit., p. 16, 18; Jérôme Pélisse, "A-t-on conscience du droit? Autour des Legal Consciousness Studies», Genèses, n 59, juin 2005, p. 114-130.

38. Iris Agmon, Family and Court: Legal Culture and Modernity in Late Ottoman Palestine, Syracuse, Syracuse University Press, 2006. 
diplomates et fonctionnaires français n'avaient d'ailleurs pas une lecture homogène du droit et des champs juridiques. À Tunis, le personnel français des tribunaux était en lutte contre la Résidence générale, représentant l'État français dans le pays; les magistrats pouvaient refuser en cette période d'appliquer les lois locales et ne voulaient reconnaître que celles de l'administration française ${ }^{39}$. Hors de ce contexte tunisien, les diplomates percevaient les tribunaux toscans comme néfastes, cherchant à miner l'autorité française ${ }^{40}$. Plus largement encore, un banquier parisien tel qu'Erlanger - très engagé dans le remboursement de la dette tunisienne, dans la succession du caïd Nissim Scemama et de facto dans celle du général Husayn - établissait à sa manière des distinctions dans ce pluralisme en dénigrant la culture juridique «inférieure» des Orientaux ${ }^{41}$. Et, de son vivant, le général Husayn parlait pour sa part de manière générique, d'autorité au sens de "droit» des Francs (bukm al-Ifranj) ${ }^{42}$. Il savait par ailleurs très bien distinguer une loi musulmane (une loi de l'Islam) des lois mises en place par le gouvernement tunisien ${ }^{43}$, tout comme il parvenait très précisément à décrire le fonctionnement de la justice italienne ${ }^{44}$. Par des réifications nécessaires à leur stratégie propre, ces acteurs distinguaient donc différents champs juridiques, mais ils se situaient en deçà d'un schéma conscient de pluralité, à un niveau intermédiaire entre une hypothétique économie morale de ce que devait être, à leurs yeux, le droit et la justice, et des idées plus complexes de distinction et de compétition entre institutions judiciaires.

Insister sur ces approches distinctes ne vise pas à invalider les dynamiques mises en évidence par Lauren Benton ou Paolo Sartori. Ici, l'objectif consiste à combiner ces deux types d'interprétation afin d'expliquer à la fois la capacité des autorités françaises à l'emporter sur le fond (en faisant reconnaître le gouverneur de Tunis comme principal héritier du général Husayn) et la validité des stratégies des particuliers qui aboutirent à des formes de reconnaissance sanctionnées par des compromis contractuels. L'accent mis sur la dynamique de mise en ordre coloniale, ou à l'inverse l'insistance sur la dextérité de "subalternes» à faire entendre leurs revendications, ne se contredisent pas. Dans le même temps, elles s'avèrent insuffisantes : elles n'épuisent pas à elles seules les dynamiques engendrées par les situations de pluralisme juridique. D'autant plus qu'il peut s'avérer intéressant, dans une troisième étape de cette réflexion, de déborder du cadre juridique et de prendre en compte d'autres domaines et d'autres institutions, élaborant d'autres types de normes.

39. Ali Noureddine, «Opposants et serviteurs...», art. cit., p. 26.

40. ANT, C11, d. 113b, doc. 9205, Santillana au secrétaire général, Régnault.

41. ANT, C. 11, d. 98, doc. 17, Erlanger à Guttieres, Paris, 4 octobre 1889 .

42. ANT, C. 11 d 105 doc. 7730 (2118) Husayn à Mustafā Murālī, 19 rajab 1299, 6 juin 1882.

43. ANT, C. 11, d. 97 doc. 86, Guttieres à Bompard, secrétaire général du gouvernement tunisien, Livourne, 12 octobre 1883; ANT, C. 11, d. 97, doc. 209, réponse à la réplique de M. Guttieres, Nice, 4 août 1885).

44. ANT, C. 11, d. 109, doc. 7989, général Husayn au Premier ministre tunisien, sans date. 


\section{LES DIFFÉRENTS REGISTRES DE NORMATIVITÉ}

Au fondement de la notion de pluralisme juridique, l'idée que le droit est davantage qu'un droit impulsé par l'État ("Law is more than State Law») permet d'explorer d'autres institutions et d'autres secteurs producteurs de normes non juridiques, ou qui dupliquent par leurs formes et par leurs fins des normes juridiques ${ }^{45}$. Dans l'affaire de la succession du général Husayn, deux autres domaines de production de normes ${ }^{46}$ peuvent être pris en compte pour mieux saisir les dynamiques engendrées par la cristallisation autour du droit en situation de pluralisme juridique : il s'agit du service rendu à l'autorité administrative et des finances publiques et privées.

Les conflits qui émergent autour de cette succession ont beaucoup à voir avec les mutations des formes et des conditions du service de gouvernement dans la province ottomane de Tunis au cours du XIX siècle. À partir des années 1830, à partir de ce qu'il est convenu d'appeler le temps des réformes ottomanes (ou tanzìmāt) de l'armée, de la fiscalité et des administrations, ce service est régi par deux registres normatifs dont les termes sont renouvelés et l'équilibre repensé. Des valeurs et des normes d'obéissance liées à la fidélité personnelle, aux pratiques de patronage, côtoient d'une nouvelle manière des normes de relation interpersonnelle et de service plus contractuelles et plus rigides. De façon plus nette que par le passé, émergent alors des règlements de service, des contrats d'engagement, des hiérarchies formelles dans les administrations civiles et militaires. Une difficulté majeure posée par la reconfiguration de ce double registre de l'exercice et de la conception du service a, par exemple, été illustrée dans les années 1880, par les poursuites que l'autorité tunisienne sous protectorat tunisien voulait intenter à l'encontre du général Husayn. Celui-ci était accusé d'avoir outrepassé les pouvoirs qui lui avaient été confiés durant sa mission jusqu’à présenter des comptes suspects. Le général clamait sa bonne foi, et se concevait comme un serviteur cherchant à défendre l'intérêt de son pays d'origine par tous les moyens ${ }^{47}$. Après son décès, les attaques et procès multiples initiés par son bras droit, Léon Elmilik, peuvent aussi s'interpréter par des décalages de perceptions de ce que devait être le service rendu à une autorité politique. Dans sa correspondance, ses mémoires et plaidoyers, Elmilik invoquait, pour défendre son bon droit, à la fois sa fidélité personnelle à toute épreuve envers le général et les promesses de son employeur.

45. Les relations entre ces ordres légaux ont suscité plusieurs lectures théoriques pensant soit ces ordres comme séparés au sein d'un même espace politique ou comme interpénétrés (Beaudoin Dupret, "Legal Pluralism, Plurality of Laws...', art. cit., p. 8).

46. Au sens de règles implicites ou parfois explicites, portés à l'écrit sous une forme contractuelle ou non contractuelle.

47. ANT, C. 11, d. 97, doc. 209, Husayn à Guttieres, Nice, 4 août 1885, le général se présente comme "un membre du gouvernement lui même élu par le conseil des ministres", "abandonné avec pleins pouvoirs». 
De ce point de vue, ces conflits n'éclairaient pas un mode d'établissement d'un ordre ou d'un désordre colonial, mais une dynamique plus ancienne d'édification étatique dans un contexte provincial ottoman dont les legs et les effets continuaient à se faire sentir dans un moment de transition et d'éloignement de la tutelle et de la culture administrative ottomanes dans cette région. Le cadre d'analyse colonial étant souvent privilégié dans les études du Maghreb, ces legs ont été sous-estimés, ou perçus au mieux comme des vestiges, au pire comme des excentricités sans réel impact sur les conceptions et les pratiques locales, sociales et politiques.

Mais ce temps des réformes, comme nous l'avons déjà vu, et cette complexification du service rendu au prince ont aussi eu un impact financier. Les réformes du service princier ont coûté cher du fait des nouvelles infrastructures mais aussi des compensations financières qu'elles induisaient, car à défaut de garantir ou d'obtenir une fidélité personnelle dans des périodes de hiérarchisation plus formelle, il a fallu monnayer les obéissances et les allégeances. De ce point de vue, les questions de finances publiques et privées ont constitué un autre domaine de production de normes, également lié au juridique, qui aide à mieux comprendre ce que ce cas d'étude révèle de rapports de force sociaux et de dynamiques historiques. L'importance croissante du lien financier, notamment quand ce type de relation était contractualisé, a rigidifié les relations d'obligations d'un débiteur envers son créancier. Dans l'ancienne province ottomane de Tunis et en Toscane, ces relations de crédits étaient observées au sein de plusieurs groupes sociaux : parmi les communautés rurales astreintes à payer des taxes ou des amendes collectives; parmi des dignitaires ne sachant comment valoriser leurs investissements dans le foncier; et dans le conflit autour de la succession du général Husayn, parmi les plaignants endettés auprès d'avocats, parmi les locataires de terres du défunt général ou parmi les dignitaires tenus par des créances auprès de financiers européens... D’une manière comparable à ce que révélaient les mutations du service princier, ces réseaux financiers nourrissaient des relations plus contraignantes que par le passé, des relations formées donc avant le temps colonial, qui avaient contribué à l'instauration du protectorat français. Ce tissu densifié de l'endettement, les mutations parallèles du service princier, les effets d'un contrôle colonial sur les institutions judiciaires, toutes ces dynamiques percent dans ce conflit de succession, et le resserrement sur les institutions judiciaires ne permet d'en révéler que quelques pans.

Or on pourrait encore ajouter les domaines de la famille ou de la piété, eux-mêmes porteurs de normativité, pour comprendre certains des aspects de la succession Husayn, mais en s'en tenant aux dynamiques de conflictualité autour du droit, se posent à la fois le problème des relations entre ces sphères normatives, de leur cohérence d'ensemble, et surtout des problèmes méthodologiques d'une réflexion autour d'études de cas par des archives judiciaires qui obligent à mettre de côté d'autres domaines de production des normes 
et les corpus qui peuvent leur être liés. Prendre connaissance des apports et des limites de l'outil conceptuel que constitue le pluralisme juridique peut révéler des horizons d'analyse pour l'étude de ce cas, mais cela pose aussi la question des dimensions ou de l'échelle de l'étude. Les archives liées à un cas judiciaire peuvent bien sûr être lues pour y collecter des informations mais aussi comme actions en elles-mêmes : au sens où les sources sont produites dans un contexte spécifique, qui, certes, les affectent mais sur lequel, surtout, elles agissent en retour ${ }^{48}$. Cependant, ces archives témoignent de différents types de relations entre sphères normatives : elle peuvent indiquer un réseau dense de relations ou à l'inverse des marques d'autonomie entre ces sphères.

Tout en étant discuté notamment pour son européo-centrisme, ce concept de "pluralisme juridique» aide à la fois à historiciser des dynamiques de rencontres juridiques et à pointer des limites dans des choix méthodologiques. En partant de ce que des archives laissent transparaître des conceptions même du droit, c'est aussi un temps d'élaboration de cette notion de pluralisme juridique que l'on historicise, en mettant en valeur l'influence que les juristes eurent à la fois sur les représentations que leurs clients avaient des institutions judiciaires, sur la judiciarisation voire la nationalisation de champs normatifs, ou bien encore sur les perceptions évolutionnistes et culturalistes des sociétés et du droit à cette époque.

Là encore, en essayant de se demander pourquoi plusieurs types d'acteurs interviennent et recourent à plusieurs degrés judiciaires dans une affaire transnationale, on peut aussi tenter de comprendre comment des cristallisations autour du droit et de conflits juridiques à un moment historique donné ont été rendues possibles, autant par l'essentialisation d'un champ normatif musulman en droit musulman que par des ramifications plus complexes et plus resserrées de statuts sociaux, de patrimoines et de hiérarchies judiciaires entre plusieurs territoires donnés. Cette situation peut alors aider à saisir comment, par ces cristallisations, des discours juridiques alternatifs à la portée potentiellement subversive ont été évacués ${ }^{49}$; comment une mise en rapport plus intense de groupes sociaux par le droit a abouti à lisser des différences, ou à penser la pluralité des institutions et du droit en fonction d'un rapport de forces et avant tout au miroir des expériences européennes.

D'un point de vue plus méthodologique, rappeler que cette pluralité juridique se reconfigure en un contexte large de transition impériale et que sa conceptualisation est énoncée a posteriori, rappeler que, dans ces analyses,

48. Simona Cerutti, «"À rebrousse-poil” : dialogue sur la méthode», Critique, 769-770, juinjuillet 2011, p. 572.

49. Simon Roberts, 'Against Legal Pluralism...', art. cit., p. 96 : Boaventura de Sousa Santos a développé l'idée d'un pluralisme juridique qui permet de redécouvrir le «pouvoir subversif de discours supprimés". 
une part trop belle a été faite aux situations coloniales ou pour le moins à des cadres d'analyse coloniaux et nationaux, peut aussi amener à prendre de la distance avec LE colonial et à inclure ce cadre parmi d'autres «bassins d'historicité» à cette échelle régionale ${ }^{50}$. Car le recours au concept de "pluralisme juridique» a montré que l'ordre juridique colonial fut en construction constante, par négociation permanente et contrainte de compromis, tant les rivalités impériales et les complexités des régimes d'appartenance étaient encore prégnantes. Plus encore, la cristallisation autour de conflits juridiques, dans la période que nous traitons, souligne à quel point d'autres espaces juridiques et d'autres dynamiques rivalisaient avec ceux qui furent établis par des autorités coloniales. Ces autres espaces, dynamiques et legs furent à la fois les fondements et les enjeux souterrains de conflits traduits, entre autres, dans le langage juridique.

M'hamed Oualdi est Assistant professor à l'Université de Princeton

50. Romain Bertrand, "Rencontres impériales. L'histoire connectée et les relations euroasiatiques", Revue d'Histoire Moderne et contemporaine, n 54-4 bis, 2007, p. 82. 